\title{
SESQUITERPENOS DO CAULE DE Pilocarpus riedelianus E ATIVIDADES SOBRE MICROORGANISMOS
}

\author{
Gisleine Guerreiro, Janete Eliza S. de Lima, João Batista Fernandes*, M. Fátima das G. F. da Silva e Paulo Cezar Vieira \\ Departamento de Química, Universidade Federal de São Carlos, CP 676, 13560-970 São Carlos - SP \\ Claudia Maria I. Magalhães, Fernando Carlos Pagnocca, Odair Corrêa Bueno e Maria José Aparecida Hebling \\ Centro de Estudos de Insetos Sociais, Instituto de Biociências, Universidade Estadual Paulista, Av. 24-A, 1515, \\ 13506-900 Rio Claro - SP \\ José Rubens Pirani \\ Departamento de Botânica, Instituto de Biociências, Universidade de São Paulo, CP 11461, 05422-970 São Paulo - SP
}

Recebido em 25/8/04; aceito em 14/3/05; publicado na web em 24/8/05

\begin{abstract}
SESQUITERPENES FROM THE STEM OF Pilocarpus riedelianus AND ANTIMICROBIAL ACTIVITY. Pilocarpus riedelianus was studied in order to obtain compounds with activity against fungi and bacteria. The dichloromethane extract, the most active one, was chromatographed yielding hexane and dichloromethane fractions. Six known sesquiterpenes, $\alpha$-calacorene, $\beta$-calacorene, $\gamma$-calacorene, cadalene, sesquichamaenol and 1-hydroxy-1,3,5-bisabolatrien10 -one were identified in the hexane fractions. The identification of these compound was done by NMR and GC/MS analyses. The hexane fraction from the dichloromethane extract showed activity against several fungi and bacteria.
\end{abstract}

Keywords: Pilocarpus riedelianus; sesquiterpenes; antimicrobial activity.

\section{INTRODUÇÃO}

A família Rutaceae tem se distinguido pela sua alta variedade de metabólitos secundários, tais como alcalóides, cumarinas, lignanas e terpenóides ${ }^{1}$. Estas classes de compostos apresentam grande variedade de atividade biológica, como por exemplo antifúngica, bactericida, antiviral e insetida ${ }^{2}$. Rutaceae tem sido alvo de estudo contínuo pelo grupo de produtos naturais do Departamento de Química da Universidade Federal de São Carlos, incluindo um estudo anterior do caule de outro espécime de Pilocarpus riedelianus ${ }^{3}$.

Pilocarpus riedelianus (Engler, Rutaceae) é uma espécie subarbustiva, natural da América Tropical. Dentre os constituintes majoritários isolados de Pilocarpus, que são conhecidos popularmente por jaborandis ${ }^{4}$, estão cumarinas, alcalóides imidazólicos e terpenóides ${ }^{5}$.

O objetivo deste trabalho foi relatar os resultados dos ensaios realizados com microorganismos em presença dos extratos do caule de $P$. riedelianus e o isolamento dos sesquiterpenos presentes no extrato mais ativo.

\section{PARTE EXPERIMENTAL}

\section{Instrumentações e procedimentos gerais}

A análise por CG/EM foi obtida em equipamento Shimadzu QP5000, coluna capilar DB-5 poli (fase: 5\% difenil, 95\% dimetilsiloxano), dimensão (15 $\mathrm{m}$ x 0,25 mm, 0,25 $\mu \mathrm{m}$ de filme), detector por ionização em chama, hidrogênio como gás carreador (1,5 mL/min). Condições cromatográficas: programação de temperatura de 60 a $280{ }^{\circ} \mathrm{C}$, utilizando rampa: $60{ }^{\circ} \mathrm{C}$ (permanência: 2 min), 60 a $240{ }^{\circ} \mathrm{C}$ com variação de $5{ }^{\circ} \mathrm{C} / \mathrm{min}$ (permanência: $5 \mathrm{~min}$ ); 240 a $280{ }^{\circ} \mathrm{C}$ com variação de $10{ }^{\circ} \mathrm{C} / \mathrm{min}$ (permanência: $5 \mathrm{~min}$ ), temperatura do injetor: $225^{\circ} \mathrm{C}$, temperatura do detector: $280{ }^{\circ} \mathrm{C}$, injeção de $1 \mu \mathrm{L}$ a uma concentração de 100 ppm de amostra.
Os espectros de $\mathrm{RMN}{ }^{1} \mathrm{H}$ e ${ }^{13} \mathrm{C}$ foram registrados em aparelho Bruker DRX 200, utilizando $\mathrm{CDCl}_{3}$ como solvente e TMS como padrão interno.

\section{Material vegetal}

O caule de $P$. riedelianus foi coletado no município de Ilhéus BA, no mês de fevereiro de 1993. A espécie foi identificada pelo Dr. J. R. Pirani e uma espécie testemunha (81627) da mesma foi depositada no herbário do Departamento de Botânica do Instituto de Biociências da USP - São Paulo, Brasil.

\section{Microorganismo}

Para os ensaios com microorganismos foi utilizado o método de difusão em ágar, utilizando discos de papel de filtros de $6 \mathrm{~mm}$ de diâmetro. Os extratos e frações foram classificados quanto à sua atividade antimicrobiana, comparando os diâmetros dos halos de inibição com o do fungicida nistatina e do bactericida cloranfenicol (controles $)^{6-8}$. Os discos controles foram embebidos com os respectivos solventes e tratados da mesma forma que os discos contendo os extratos. Os discos foram submetidos ao processo de secagem em estufa para evaporação do solvente. Os meios utilizados nos ensaios foram Ágar Sabouraud para os fungos e Ágar Nutriente para as bactérias. Para preparação do inoculo utilizou-se uma suspensão de células em solução fisiológica $0,85 \%$, a qual foi padronizada pela Escala de Mc Farland $\mathrm{n}^{\circ} 0,5$. Uma alíquota de $100 \mu \mathrm{L}$ dessa suspensão foi espalhada na superfície do meio com "swab" estéril. Os discos foram depositados na superfície do meio e levemente pressionados sobre o ágar. Foi mantida uma distância de $1,5 \mathrm{~cm}$ entre os discos e de $1 \mathrm{~cm}$ da borda para evitar sobreposição dos halos de inibição. Após a semeadura, as placas foram invertidas e incubadas por $24 \mathrm{~h}$ à $35^{\circ} \mathrm{C}$ no caso das bactérias e $48 \mathrm{~h}$ à $25^{\circ} \mathrm{C}$. Foi realizada a leitura dos resultados medindose o diâmetro dos halos de inibição. Cada ensaio foi realizado em duplicata e repetido pelo menos uma vez ${ }^{7}$. 


\section{Extração, fracionamento e identificação dos compostos de $P$. riedelianus}

O caule de $P$. riedelianus $(4,8 \mathrm{~kg})$ foi moído e em seguida extraído por percolação durante 3 dias e com 3 repetições. Na sequiência de extração foram utilizados os solventes hexano, diclorometano e metanol, originando os respectivos extratos hexânico (21,9 g), diclorometânico (23,6 g) e metanólico (109,5 g).

O extrato diclorometânico $(23,6 \mathrm{~g})$ foi fracionado utilizando-se cromatografia rápida à pressão reduzida, usando gel de sílica e solventes puros em ordem crescente de polaridade. Foram obtidas as frações hexânica $(0,49 \mathrm{~g})$, diclorometânica $\mathrm{A}(3,35 \mathrm{~g})$, diclorometânica $\mathrm{B}(0,79$ g), acetoetílica $(5,96 \mathrm{~g})$ e metanólica $(1,13 \mathrm{~g})$. Por apresentar atividade sobre os microorganismos, a fração hexânica foi selecionada para refracionamento através de coluna cromatográfica usando gel de sílica (230-400 mesh) e sistema gradiente de solvente, iniciando com hexano, seguido por acetato de etila e encerrando com metanol. Foram obtidas 7 subfrações, sendo que as subfrações 1 e 3 foram novamente refracionadas, nas mesmas condições da anterior, originando 7 subfrações em ambas colunas (1-1 a 1-7 e 3-1 a 3-7). As subfrações 14 (3,2 mg) e 1-5 (2,3 mg) do fracionamento da fração original 1 continham, predominantemente, os sesquiterpenos sesquichamaenol (5) e 1-hidroxi-1,3,5-bisabolatrien-10-ona (6), respectivamente. $\mathrm{Na}$ subfração 3-7 da fração original 3 foram identificados os sesquiterpenos 1-6 em mistura (1,6 mg) através de CG/EM.

\section{RESULTADOS E DISCUSSÃO}

A análise do extrato diclorometânico do caule de $P$. riedelianus levou ao isolamento de seis sesquiterpenos (1-6). Foram identificados quatro sesquiterpenos cadinanos (1-4), um bisabolano (6) e sesquichamaeno (5), cuja ocorrência é restrita a poucas famílias vegetais. Os compostos $\alpha$-calacoreno $(\mathbf{1})^{9-12}, \beta$-calacoreno $(\mathbf{2})^{9}, \gamma$-calacoreno $(3)^{10-12}$, cadaleno (4) $)^{10-12}$ sesquichamaenol (5) $)^{13-17}$ e 1-hidroxi-1,3,5bisabolatrien-10-ona (6) ${ }^{15,17}$ foram identificados através de seus dados de RMN ${ }^{1} \mathrm{H}$ e ${ }^{13} \mathrm{C}$, CG-EM (Figuras 1-3) em comparação aos relatados na literatura.

Nos espectros de RMN ${ }^{13} \mathrm{C}$ (Tabela 1) das subfrações 1-4 e 1-5 foram observados, respectivamente, os sinais para carbonos carbonílicos $\delta$ 210,7 e 217,5, assim como 6 sinais para carbonos aromáticos e outros 9 sinais para carbonos alifáticos, completando 15 carbonos para cada componente das subfrações, indicando tratarem de sesquiterpenos. O espectro de RMN ${ }^{1} \mathrm{H}$ da subfração 1-4 (fr 1-4) apresentou sinais característicos de hidrogênio em sistema aromático 1,2,4-trissubstituído: $\delta$ 6,83 (sl), 6,86 (dd, 8,0 e 1,6 Hz) e 6,67 (d, 8,0 $\mathrm{Hz}$, grupo isopropila: $\delta 0,73\left(\mathrm{~d}, 6,8 \mathrm{~Hz}, \mathrm{CH}_{3}\right), 1,01\left(\mathrm{~d}, 6,8 \mathrm{~Hz}, \mathrm{CH}_{3}\right)$ e $1,82(\mathrm{~m}, \mathrm{CH})$, metila alfa à carbonila: ( $\mathrm{s}$ e metila em anel aromático: $\delta$ 2,25 (s). O espectro de RMN ${ }^{1} \mathrm{H}$ da fração 1-5 (fr 1-5) (Tabela 1) mostrou-se semelhante ao da subfração $1-4$, porém não apresentou sinal de hidrogênio de metila alfa à carbonila, sendo substituído por um dubleto em $\delta 1,25(\mathrm{~J}=6,8 \mathrm{~Hz})$, também correspondente a hidrogênios de um grupo metílico. Além disso, o deslocamento químico do hidrogênio do grupo metínico da isopropila $(2,90, \mathrm{~m})$ sugere vizinhança à carbonila. A comparação dos dados de $\mathrm{RMN}{ }^{1} \mathrm{H}$ e ${ }^{13} \mathrm{C}$ do sesquiterpeno da subfração 1-4 com os do sesquichamaenol (5) isolado anteriormente de Chamaecyparis formosensis Matsum (Cupressaceae) $)^{13}$, Cedrolopsis microfoliata J. F. Leroy (Ptaeroxylaceae) ${ }^{14}$ e de Juniperus formosan Hay. var. concolor Hay (Cupressaceae $)^{15}$ possibilitou comprovar que se tratava da mesma substância, identificando assim o principal constituinte desta subfração. $\mathrm{O}$ sesquichamaenol tem origem a partir do calacoreno através de reação de clivagem oxidativa. Neste caso, a clivagem ocorreu entre os carbonos 1 e 9, mas poderia ocorrer também entre 4 e 10. Com base nessa
Tabela 1. Dados de $\mathrm{RMN}{ }^{1} \mathrm{H}$ e ${ }^{13} \mathrm{C}\left(200 \mathrm{MHz}, \mathrm{CDCl}_{3}, \delta, J \mathrm{~Hz}\right)$ para sesquichamaenol (5) ${ }^{14-17}$ e 1-hidroxi-1,3,5-bisabolatrien-10-ona $(6)^{15,17}$

\begin{tabular}{|c|c|c|c|c|}
\hline \multirow[t]{2}{*}{$\mathrm{C}$ ou $\mathrm{H}$} & \multicolumn{2}{|c|}{$\begin{array}{l}\text { Sesquichamaenol* } \\
\quad(\text { fr } 1-4)\end{array}$} & \multicolumn{2}{|c|}{$\begin{array}{l}\text { 1-hidroxi-1,3,5- } \\
\text { bisabolatrien-10-ona* } \\
\text { (fr } 1-5)\end{array}$} \\
\hline & $\mathrm{RMN}{ }^{1} \mathrm{H}$ & $\mathrm{RMN}{ }^{13} \mathrm{C}$ & $\mathrm{RMN}{ }^{1} \mathrm{H}$ & $\mathrm{RMN}{ }^{13} \mathrm{C}$ \\
\hline 1 & & 210,7 & $1,70 \mathrm{~m}$ & 31,8 \\
\hline 2 & $2,10 \mathrm{~m}$ & 41,8 & $1,60 \mathrm{~m}$ & 30,5 \\
\hline 3 & $1,80 \mathrm{~m}$ & 26,7 & $1,70 \mathrm{~m}$ & 40,9 \\
\hline $\mathrm{dd}$ & $\begin{array}{c}2,58 \\
1,11,6 ; 8,4 \text { e } 3\end{array}$ & 44,1 & & 217,5 \\
\hline 5 & $6,83 \mathrm{sl}$ & 128,5 & $6,70 \mathrm{sl}$ & 116,9 \\
\hline 6 & & 130,0 & & 137,1 \\
\hline 7 & $\begin{array}{c}6,86 \\
\text { dd, } 8,0 \text { e } 1,6\end{array}$ & 127,3 & $\begin{array}{c}6,69 \\
\mathrm{~d}, 8,0\end{array}$ & 121,0 \\
\hline 8 & $\begin{array}{c}6,67 \\
\mathrm{~d}, 8,0\end{array}$ & 115,7 & $\begin{array}{c}7,00 \\
\mathrm{~d}, 8,0\end{array}$ & 125,7 \\
\hline 9 & & 151,9 & & 128,7 \\
\hline 10 & & 129,8 & & 154,0 \\
\hline 11 & $1,82 \mathrm{~m}$ & 33,0 & $2,90 \mathrm{~m}$ & 37,5 \\
\hline 12 & $\begin{array}{c}0,73 \\
d, 6,8\end{array}$ & 21,3 & $\begin{array}{c}1,08 \\
d, 6,8\end{array}$ & 18,3 \\
\hline 13 & $\begin{array}{c}1,01 \\
\mathrm{~d}, 6,8\end{array}$ & 21,0 & $\begin{array}{c}1,10 \\
\mathrm{~d}, 6,8\end{array}$ & 18,3 \\
\hline 14 & $2,05 \mathrm{~s}$ & 30,1 & $\begin{array}{c}1,25 \\
d, 6,8\end{array}$ & 18,9 \\
\hline 15 & $2,25 \mathrm{~s}$ & 20,8 & $2,27 \mathrm{~s}$ & 20,7 \\
\hline
\end{tabular}

* numeração dos carbonos de acordo com o sesquiterpeno de origem biossintética mostrada nas estruturas e nomes de acordo com os já citados na literatura para 5-(2-hidroxi-5-metilfenil)-6-metil-hepten2-ona $=$ sesquichamaenol (5) e 6-(2-hidroxi-5-metilfenil)-2-metilhepten-3-ona $=1$-hidroxi-1,3,5-bisabolatrien-10-ona $(\mathbf{6})$.

hipótese comparam-se os dados de $\mathrm{RMN}{ }^{1} \mathrm{H}$ e ${ }^{13} \mathrm{C}$ (Tabela 1) da subfração 1-4 (sesquichamaenol) e da subfração 1-5, que contêm um composto com a mesma massa molecular do sesquichamaenol, $\mathrm{m} / \mathrm{z}$, 234 Daltons. Esta análise e a comparação com os dados de RMN de ${ }^{1} \mathrm{H} \mathrm{e}{ }^{13} \mathrm{C}$ relatados para 1-hidroxi-1,3,5-bisabolatrien-10-ona $(6)^{15,17}$, permitiram identificá-lo na subfração 1-5. As Figuras 4 e 5 mostram as propostas de fragmentações no espectrômetro de massas para 5 e 6 e os íons fragmentários $\mathrm{m} / \mathrm{z} 148(98 \%)$ e 135 (100\%) Daltons para 6 e $133(100 \%)$ e $121(80 \%)$ Daltons para 5, que permitem diferenciar as estruturas destes sesquiterpenos por EM.

Os sesquiterpenos 1 a 6 foram identificados na subfração 3-7. A análise do espectro de $\mathrm{RMN}{ }^{13} \mathrm{C}$ desta fração permitiu verificar sinais nas regiões de carbonos aromáticas e alifáticas com deslocamentos químicos semelhantes aos observados para $\mathbf{5}$ e $\mathbf{6}$. O número de sinais próximos entre si e as intensidades dos mesmos eram indicativos que esta subfração continha mistura de sesquiterpenos estruturalmente semelhantes. A identificação dos constituintes da mistura foi realizada pela análise em CG/EM (Figura 1) e comparação de seus espectros de massas (Figuras 2 e 3 ) com aqueles da biblioteca NIST-62, contida no computador do espectrômetro de massas, assim como com seus tempos de retenções. Para os picos 1 a 3 (Figuras 1 e 2, espectros 1-3), foram identificados $\alpha$-calacoreno (1), $\beta$-calacoreno (2) e $\gamma$-calacoreno (3) e para os picos 4-6 (Figura 1 e 3, espectros 4-6), respectivamente cadaleno (4), sesquichamaenol (5) e 1-hidroxi-1,3,5-bisabolatrien-10ona (6).

De acordo com os resultados dos ensaios microbiológicos apresentados na Tabela 2, a fração hexânica do extrato diclorometânico apresentou o melhor resultado, sendo ativa para a maioria dos 
microorganismos testados, inclusive para Candida krusei, que produz metabólitos tóxicos durante processo infeccioso ${ }^{18}$, os quais podem ser fatais, em especial para indivíduos imunodeficientes.

A comparação com estudos realizados anteriormente por Trotti ${ }^{19}$ sobre a ação dos extratos do caule de Pilocarpus riedelianus e suas frações sobre microorganismos, verificou que a fração hexânica do extrato hexânico apresentou-se como a mais ativa. Esta fração era constituída pela mistura de 1-hidroperoxicalameneno, 1hidroxicalameneno e sesquichamaenol. Neste trabalho, sesquichamaenol estava presente em mistura juntamente com outros sesquiterpenos na fração hexânica do extrato diclorometânico de $P$. riedelianus, que mostrou atividade em diversos microorganismos (Tabela 2), porém não foram isolados desta fração o 1hidroperoxicalameneno e 1-hidroxicalameneno. Esta observação sugere que o sesquichamaenol possa ser o responsável pelas atividades apresentadas pela fração hexânica sobre Staphylococcus aureus, Micrococcus roseus, Bacillus subtilis, Bacillus cereus, Salmonella scholeraesuis, Candida krusei, Cryptococcus laurentii, Rhodotorula rubra e Saccharomyces cerevisiae ou, ainda, que os sesquiterpenos atuem sinergisticamente, potencializando sua ação antimicrobiana.<smiles>CC1=C[C@H]2C(C=C1)C(N)=CC[C@@H]2C(C)C</smiles>

(1)<smiles>Cc1ccc2c(C)ccc(C(F)(F)F)c2c1</smiles>

(4)
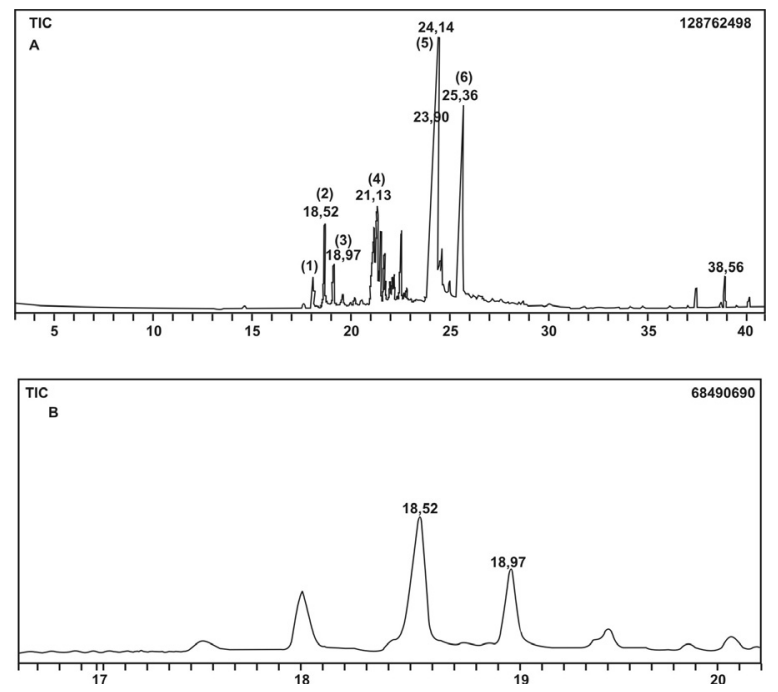

Figura 1. Cromatograma dos íons totais (TIC) da subfração HDPr3-7: $\alpha$-calacoreno (1), $\beta$-calacoreno (2), $\gamma$-calacoreno (3); cadaleno (4); sesquichamaenol (5) e 1-hidroxi-1,3,5-bisabolatrien-10-ona (6). A: TIC total; B: expansão do TIC no intervalo de 17-20 min
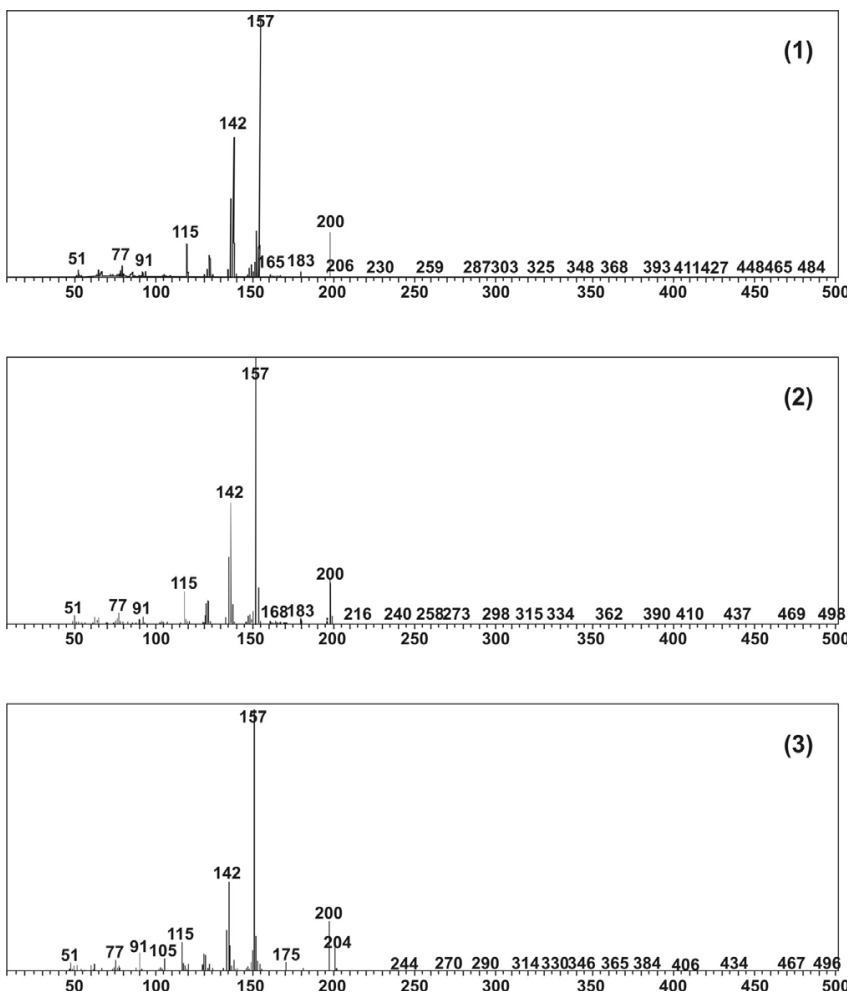

Figura 2. Espectros de massas da subfração HDPr3-7, dos picos correspondentes a $\alpha$-calacoreno (1), $\beta$-calacoreno (2) e $\gamma$-calacoreno (3), respectivamente
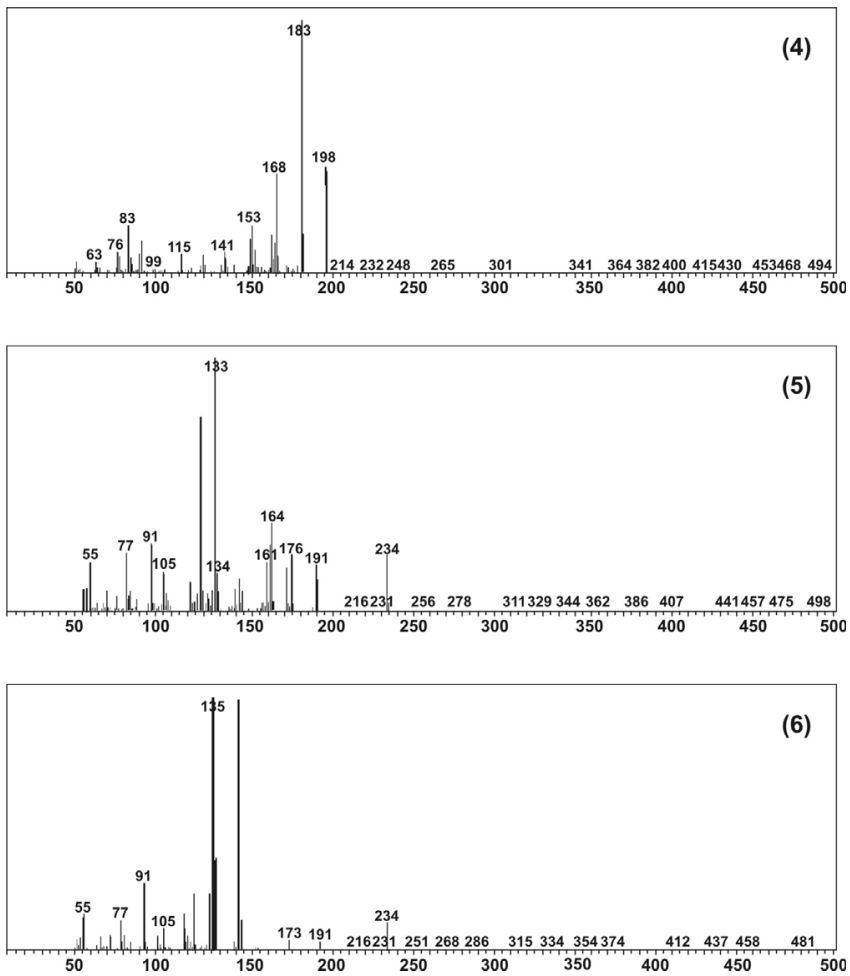

Figura 3. Espectros de massas da subfração HDPr3-7, dos picos correspondentes ao cadaleno (4), sesquichamaenol (5) e 1-hidroxi-1,3,5bisabolatrien-10-ona (6) 
Tabela 2. Diâmetro (mm) dos halos de inibição do extrato diclorometânico do caule de Pilocarpus riedelianus (DPr) e suas frações hexânica (DHPr), diclorometânica (DDPr) e acetoacetilica (DAcPr) sobre bactérias e fungos

\begin{tabular}{|c|c|c|c|c|c|}
\hline Microorganismo/Extrato ou Fração & DPr & DHPr & DDPr & $\mathrm{DAcPr}$ & Controle $^{\mathrm{A}}$ \\
\hline Staphylococcus aureus ${ }^{\mathrm{B}}$ ATCC/6538 & 14 & 12 & 11 & 9 & 23 \\
\hline Micrococcus roseus ${ }^{\mathrm{B}}$ CCT/1469 & Traço & 16 & 10 & - & 49 \\
\hline Bacillus subtilis ${ }^{\mathrm{B}} \mathrm{CCT} / 2471$ & Traço & 13 & - & - & 25 \\
\hline Bacillus cereus ${ }^{\mathrm{B}} \quad \mathrm{CCT} / 1436$ & 10 & 11 & 8 & 9 & 27 \\
\hline Pseudomonas aeruginosa ${ }^{\mathrm{B}}$ ATCC/15442 & - & - & - & - & 12 \\
\hline Pseudomonas fluorescens ${ }^{\mathrm{B}}$ CCT-3178 & - & - & - & - & 12 \\
\hline Escherichia coli ${ }^{\mathrm{B}} \mathrm{CCT} / 1457$ & - & Traço & - & - & 26 \\
\hline Enterobacter aerogenes $^{\mathrm{B}}$ CCT/2572 & - & - & - & - & 22 \\
\hline Salmonella scholeraesuis ${ }^{\mathrm{B}}$ ATCC/10708 & - & 10 & - & - & 25 \\
\hline Candida krusei ${ }^{\mathrm{C}} \mathrm{RJ} / \mathrm{P} 007$ & - & 20 & - & - & 16 \\
\hline Cryptococcus laurentii ${ }^{\mathrm{C}} \mathrm{RJ} / 50359$ & - & 9 & - & - & 18 \\
\hline Rhodotorula rubra ${ }^{\mathrm{C}} \mathrm{RJ} / 50595$ & - & 10 & & & 20 \\
\hline Geotrichum $s p^{\mathrm{C}} \mathrm{RJ} / 003$ & - & - & & & 20 \\
\hline Saccharomyces cerevisiae ${ }^{\mathrm{C}} \mathrm{CCT} / 0758$ & - & 7 & & & 20 \\
\hline
\end{tabular}

${ }^{\mathrm{A}}$ Cloranfenicol (30 $\mu \mathrm{g} /$ disco $)$ para bactérias, nistatina (10 $\mu \mathrm{g} /$ disco) para fungos ou leveduras, ${ }^{\mathrm{B}}$ bactérias, ${ }^{\mathrm{C}}$ fungos ou leveduras. (-) Ausência de halo de inibição. Concentrações dos extratos e frações $1000 \mu \mathrm{g} /$ disco.

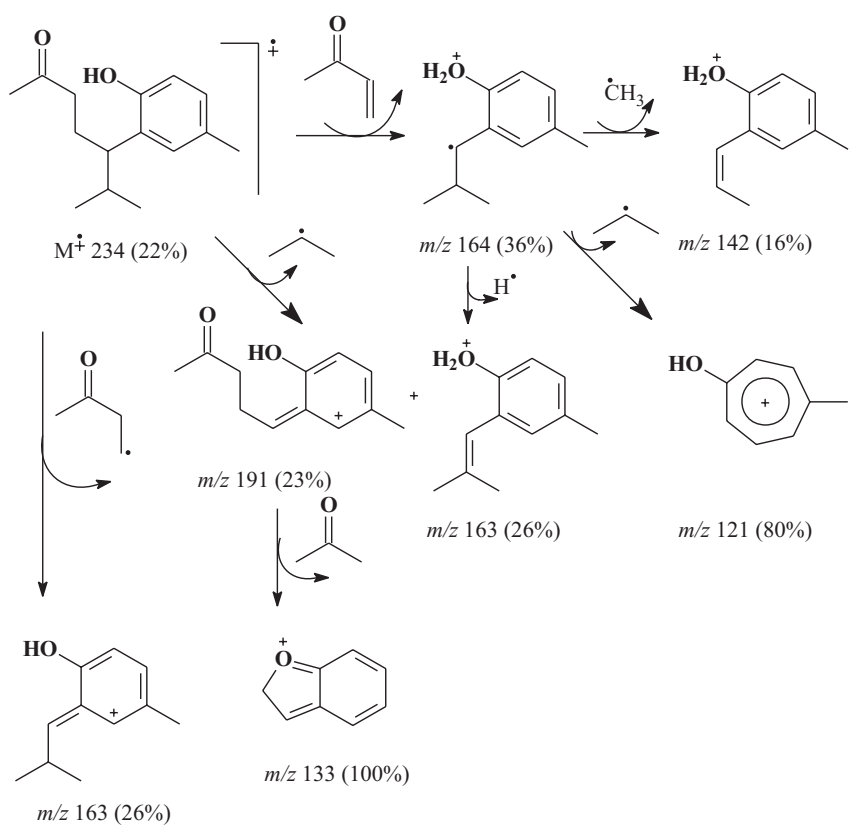

Figura 4. Fragmentograma dos principais íons obtidos no espectro de massas proposto para o sesquichamaenol (5)

Dadas as baixas concentrações isoladas dos sesquiterpenos e a importância de se obter compostos ativos contra Pesudomonas fluorescens e Enterobacter aerogenes, que são freqüentes em infecções urinárias ${ }^{6}$, somente o sesquichamaenol foi testado contra as mesmas e não mostrou atividade nas concentrações testadas: 9,5, 19,0 e $37,0 \mu \mathrm{g} / \mathrm{mL}$. Ensaios do sesquichamaenol contra os demais microorganismos, assim como dos demais sesquiterpenos deverão ser objetos de futuros estudos.

\section{AGRADECIMENTOS}

Ao CNPq, FAPESP, CAPES e PRONEX-CNPq-FINEP pelo apoio financeiro.
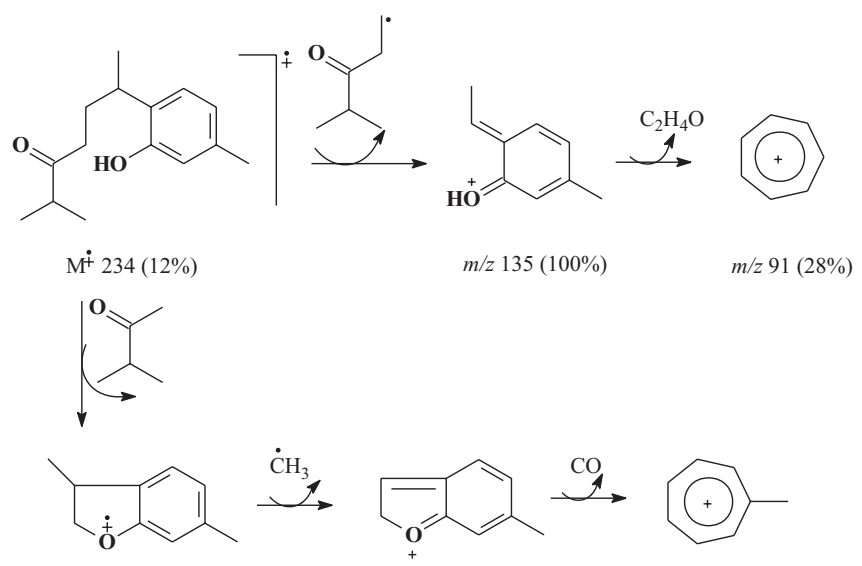

$m / z 148(99 \%)$
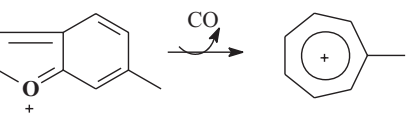

$m / z 133(22 \%)$

$m / z 105(17 \%)$

Figura 5. Fragmentograma dos principais ions obtidos no espectro de massas proposto para o 1-hidroxi-1,3,5-bisabolatrien-10-ona (6)

\section{REFERÊNCIAS}

1. da Silva, M. F. das G. F.; Gottlieb, O.R.; Ehrendorfer, F.; Plant System. Evol. 1998, 16, 97

2. Lewis, J. R. Em Chemistry and Chemical Taxonomy of Rutales; Waterman, P. G.; Grundon, M. F., eds.; Academic Press: London, 1983.

3. Ambrozin, A. R. P.; Vieira, P. C.; Fernandes, J. B.; da Silva, M. F. G. F.; Albuquerque, S.; Mem. Inst. Oswaldo Cruz 2004, 99, 227; Moraes, V. R. de S.; Tomazela, D. M.; Ferracin, R. J.; Garcia, C. F.; Sannomiya, M.; Soriano, M. del P. C.; da Silva, M. F. G. F.; Vieira, P. C.; Fernandes, J. B.; Rodrigues Fo., E.; Magalhães, E. G.; Magalhães, A. F.; Pimenta, E. F.; Souza, D. H. F.; Oliva, G.; J. Braz. Chem. Soc. 2003, 14, 380; Sartor, C. F. P.; da Silva, M. F. G. F.; Fernandes, J. B.; Vieira, P. C.; Rodrigues Fo., E.; Cortez D. A. G.; Phytochemistry 2003, 63, 185; Biavatti, M. W.; Vieira, P. C.; da Silva, M. F. G. F.; Fernandes, J. B.; Albuquerque, S.; Magalhães, S. C. M. I.; Pagnocca, F. C.; Phytomedicine 2001, 8, 121; Biavatti, M. W.; Vieira, P. C.; da Silva, M. F. G. F.; Fernandes, J. B.; Degani, A. L. G.; Cass, Q. B.; Schefer, A. B.; Ferreira, A. G.; Phytochem. Anal. 2001, 12, 64; Tomazela, D. M.; Pupo, M. T.; Passador, E. A. P.; da Silva, M. F. G. F.; Vieira, P. C.; Fernandes, J. B.; Rodrigues Fo., E.; Pirani, J. R.; Phytochemistry 2000, 55, 643; Mafezoli, J.; Vieira, P. C.; Fernandes, J. B.; da Silva, M. F. G. F.; Albuquerque, S.; J. Ethnopharmacol. 2000, 73, 335; Santos, C. S.; Januário, A. H.; Vieira, P. C.; Fernandes, J. B.; da Silva, M. F. G. F.; Pirani, J. R.; J. Braz. Chem. Soc. 1998, 9, 39; 
Ferracin, R. J.; da Silva, M. F. G. F.; Fernandes, J. B.; Vieira, P. C.; Phytochemistry 1998, 47, 393; Passador, E. A. P.; da Silva, M. F. G. F., Rodrigues Fo., E.; Fernandes, J. B.; Vieira, P. C.; Pirani, J. R.; Phytochemistry 1997, 45, 1533; Velozo, E. S.; Oliveira, D. J.; Arruda, A. C.; Vieira, P. C.; Fernandes, J. B.; da Silva, M. F. G. F. da; Caracelli, I.; Zukerman-Schpector, J. Z.; Nat. Prod. Lett. 1997, 9, 237; Müller, A. H.; Vieira, P. C.; da Silva, M. F. G. F.; Fernandes J. B.; Phytochemistry 1995, 40, 1797; Arruda, M. S. P.; Fernandes, J. B.; Vieira, P. C.; da Silva, M. F. G. F.; Pirani, J. R.; Phytochemistry 1994, 36, 1303; Muller, A. H.; Degaspari, L. R. O.; Vieira, P. C.; da Silva, M. F. G. F.; Fernandes, J. B.; Pirani, J. R.; Phytochemistry 1993, 34, 585.

4. Grieve, M. A.; A modern herbal. The medicinal, culinary, cosmetic and economic proprieties, cultivation and folklore of herbs, grasses, fungi, shrubs and trees with all their modern scientific uses, Tiger Books International: London, 1994.

5. Souza, R. C.; Tese de Doutorado, Universidade Federal de São Carlos, Brasil, 2003.

6. Gilman, A. G.; Hardman J. G.; Limbird, L. E.; The Pharmacological Basis of Therapeutics, $9^{\text {th }}$ ed., McGraw Hill: New York, 1996, p. 57.

7. Barry, A. L.; Thornsberry, C.; Manual of Clinical of Microbiology, $3^{\text {rd }}$ ed., American Society for Microbiology: Washington, 1980, p. 463
8. Bauer, A. W.; Kirby, W. M. N.; Sherris, J. C.; Turck, M.; J. Clin. Pathol. 1966, 45, 493.

9. Adams, R. P.; Identification of Essencial Oil Components by Gas Chromatography/Mass Spectroscopy, Allured Publishing Corporation: Illinois, 1995.

10. Warmers, U.; Wihstutz, K.; Bülow, N.; Fricke, C.; König W. A.; Phytochemistry 1998, 49, 1723.

11. Warmers, U.; König W. A.; Phytochemistry 1999, 52, 99

12. Bülow, N.; König W. A.; Phytochemistry 2000, 55, 141.

13. Ando, M.; Ibe, S.; Kagabu, S.; Nakagawa, T.; Asao, T.; Takase, K.; J. Chem. Soc., Chem. Commun. 1970, 1538.

14. Koorbanally, N. A.; Randrianarivelojosia, M.; Mulholland, D. A.; van Ufford, L. Q.; van den Berg, A. J. J.; J. Nat. Prod. 2002, 65, 1349.

15. Kuo, Y. H.; Yu, M. T.; Chem. Pharm. Bull. 1996, 44, 2150.

16. Singh, V.; Khurana, A.; Kaur, I.; Sapehiyia, V.; Kad, G. L.; Singh, J.; J. Chem. Soc., Perkin Trans. 1 2002, 1766.

17. Lee, C. K.; Cheng, Y. S.; J. Chin. Chem. Soc. 2001, 48, 1077

18. Trabulsi, L. R.; Microbiologia; 2a ed., Ed. Atheneu: São Paulo, 1991, p. 105.

19. Trotti, G. R.; Dissertação de Mestrado, Universidade Estadual Paulista, Brasil, 1998. 